\title{
COVID-19 Stressors and Poor Sleep Quality: The Mediating Role of Rumination and the Moderating Role of Emotion Regulation Strategies
}

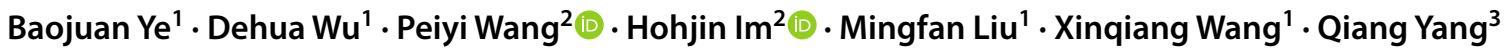

Accepted: 14 September 2021 / Published online: 18 September 2021

(c) International Society of Behavioral Medicine 2021

\begin{abstract}
Background The global spread of COVID-19 has brought immense psychological distress and sleep problems to those affected. This study examined the mediating role of rumination in the direct association between COVID-19 stressors and poor sleep quality and the moderating roles of emotion regulation strategies.

Method A cross-sectional online survey study was conducted in China during the early outbreak of the pandemic. A total of 1106 Chinese college students $\left(M_{\text {age }}=19.58, S D=1.61\right)$ completed measures of COVID-19 stressors, rumination, emotion regulation strategies (i.e., cognitive reappraisal and expressive suppression), and poor sleep quality.

Results COVID-19 stressors were positively associated with poor sleep quality $(\beta=.431, p<.001)$, and rumination partially mediated this association. The mediation effect accounted for $70.93 \%$ of the total effect of COVID-19 stressors on poor sleep quality. Moreover, cognitive reappraisal moderated the relation between COVID-19 stressors and rumination, and expressive suppression moderated the association between rumination and poor sleep quality.

Conclusion Rumination could be a mechanism by which COVID-19 stressors are linked with poor sleep quality. Cognitive reappraisal might provide desired benefits to improving sleep quality while expressive suppression may do the opposite. Implications for future steps and health interventions are discussed.
\end{abstract}

Keywords COVID-19 $\cdot$ Perceived stress $\cdot$ Rumination $\cdot$ Sleep quality $\cdot$ Emotion regulation strategy

\section{Introduction}

The COVID-19 pandemic has introduced an unprecedented number of uncontrollable stressors, negatively affecting the sleep quality of those affected [1-5]. During the early stages of the pandemic, nearly a third of residents in Wuhan,

Baojuan Ye, Dehua Wu, and Peiyi Wang share first authorship.

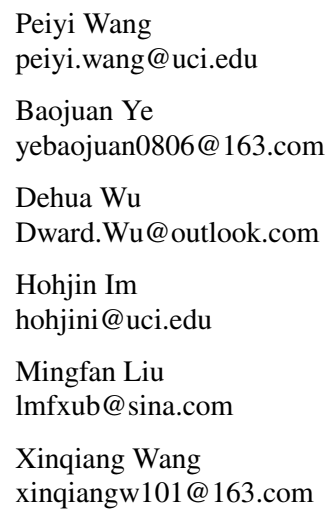

China, reported having sleep problems [6]. Another study [7] showed that $58 \%$ and $40 \%$ of participants across 49 countries, respectively, reported dissatisfaction with their sleep and poorer sleep quality compared to pre-COVID-19 times. Such statistics paint a grim picture given the importance of sleep in maintaining psychosocial wellbeing, achievement, and health condition [8-12].

Qiang Yang

davidyang12345@163.com

1 Center of Mental Health Education and Research, Center of Preschool Education, School of Psychology, Jiangxi Normal University, Nanchang, China

2 Department of Psychological Science, University of California Irvine, Irvine, CA, USA

3 School of Education, Jiangxi Normal University, Nanchang, China 
Among the studied demographic clusters, the college student population may be important to monitor during the pandemic. Typically, as young adults emerging from adolescence, college students have been reported to be highly susceptible to the negative consequences of stress, such as sleep issues [12]. Often early in their adulthood, college students may expectedly lack adequate coping skills to manage stress or engage in self-control under high-pressure situations [5, 13, 14]. Moreover, because many university campuses had shut down campuses in the wake of local outbreaks, many students were abruptly stranded without close social support or access to necessary interventions, further compounding the negative effects of stressors on the onset of sleep issues [12, 15]. Despite the wide array of COVID-19 stressors for college students [16], however, the number of investigations on how college students' own protective and risk factors influenced the path from COVID-19 stress to poor sleep quality has been sparse. The current investigation sought to fill in this gap by examining the underlying behavioral and psychological mechanisms that may assist future development of prevention and intervention efforts to sleep health.

\section{The Mediating Role of Rumination}

Rumination refers to the repetitive cognitive representations of stress in response to adverse events [17], which are typically negative and destructive in nature [18]. It represents a form of unhealthy self-focus that is activated by stressful life events [19], and induce negative stress consequences by fixating on the problems without taking action [17] and lengthening its physiological activations [20]. Expectedly, empirical studies have demonstrated the links between both COVID-19 related stress [13] and general perceived stress [21] on rumination.

Further, rumination was found to have associations with short- and long-term sleep disturbances among college students $[22,23]$ partly due to the continuous psychophysiological arousals in response to stressful life events [20]. Although studies conducted pre-COVID-19 [24] have found little evidence of the effect of rumination on sleep among individuals with little rumination disposition, recent studies have proposed that the sudden and pervasive nature of COVID-19 may develop rumination tendencies among those have previously not shown such issues [13]. Moreover, rumination served as a key mechanism in several antecedents of poor sleep quality, such as loneliness [25] and negative affect [26], both of which may be possible sources of COVID-19 related stress. Hence, rumination may mediate the association between COVID-19 stress and poor sleep quality.

\section{The Moderating Role of Emotion Regulation}

Although COVID-19 stressors may influence college students' poor sleep quality through the mediating effect of rumination, individuals might differ in their adjustments to stressful life events based on the extent to which they regulate their negative emotions [27]. Emotion regulation is the process by which individuals change the generation, expression, and intensity of emotions through strategies including cognitive reappraisal and expressive suppression $[28,29]$.

Cognitive reappraisal is an antecedent-focused strategy in which individuals reframe the interpretation of a situation with the objective to modify its emotional impact [30]. As it is the subjective experience of the event that evokes emotional responses [31,32], successful cognitive reappraisal of negative experience may relieve stressrelated difficulties and promote psychological adjustments to stressors [33]. Thus, in the context of the pandemic, students who cognitively reappraise the extent to which the pandemic may be construed as negative might have the psychological leverage in adapting to $[34,35]$ and buffering the harmful effects of stress on negative outcomes [36]. That is, cognitive reappraisal may be a mechanism dampening the effect of stress (i.e., COVID-19 related) on maladaptive consequences, such as rumination.

Expressive suppression, on the other hand, is a response-focused strategy in which one represses their emotions after the response tendencies (e.g., rumination) have been generated, such as hiding signs of distress or sadness [30]. Prior studies have found that expressive suppression may not dampen the emotional experience but rather exacerbate one's physiological responses given that it impedes emotional processing [30]. Accordingly, the heightened somatic arousal and physiological strain induced by expressive suppression have been evidenced to correlate with poorer sleep quality [37] and contribute to the onset of sleep problems [38]. Thus, the use of expressive suppression may exacerbate the effect of rumination on poor sleep quality.

\section{The Present Study}

Three aims were proposed by the current study: (a) to test whether rumination mediates the relation between COVID-19 stressors and sleep quality among Chinese college students, (b) whether the first path of the indirect relations (i.e., between COVID-19 stressors and rumination) is moderated by cognitive reappraisal, and (c) whether the second path of the indirect relations (i.e., between 
rumination and poor sleep quality) is moderated by expression suppression. As cognitive reappraisal targets reframing the event, whereas expressive suppression focuses on alternating the emotion [30], these two emotion regulation strategies were postulated to moderate these two different paths. We posited the following hypotheses:

Hypothesis 1. Rumination partially explains the positive relation between COVID-19 stressors and poor sleep quality.

Hypothesis 2. The size of the positive correlation between COVID-19 stressors and rumination is smaller among those who engage in more cognitive reappraisal than those who engage in less.

Hypothesis 3. The size of the positive correlation between rumination and poor sleep quality is larger among those who practice more expression suppression than those who practice less.

\section{Method}

\section{Procedure}

This multicenter, cross-sectional online study was conducted in Eastern China during the early stages of the COVID-19 pandemic (i.e., February to March 2020) to directly examine the study hypotheses. The questionnaire was hosted on Survey Star (an online data collection software) and distributed to participants via the social media platforms, WeChat and QQ. As the study was distributed online, students participated in the study from various regions of China. Individuals were eligible for this study if they were in the social media groups and self-identified as currently enrolled college students from the participating centers and universities. Sixtyone observations data were excluded for failing an attention check question. Because the Survey Star only recorded responses from fully completed questionnaires, there were no missing data. This study was approved by the Research Ethics Committee of the first author's institution. All participants consented to participate, and no identifiable information was collected. Participation in this study was entirely voluntary, and no compensation was given.

\section{Participants}

The final sample consisted of 1106 college students $\left(M_{\mathrm{age}}=19.58, S D=1.61\right.$, range $=18-25,57.2 \%$ female $)$. More than half $(65.3 \%)$ were 1 st years, $17.7 \%$ were 2 nd years, $9.7 \%$ were 3 rd years, and $7.3 \%$ were 4 th years. Slightly more than the majority $(54.6 \%)$ reported their place of residence as urban compared to rural.

\section{Measures}

\section{COVID-19 Stressors}

COVID-19 stressors were measured via the Stressors of COVID-19 Scale [13] in which participants rated how stressed they felt on several factors stemming from the COVID-19 pandemic. The scale is comprised of four dimensions: (1) disease stressors of COVID-19 (7 items, e.g., "the high contagiousness of COVID-19"), (2) information stressors of COVID-19 (4 items, e.g., "negative news about COVID-19"), (3) measures stressors of COVID-19 (4 items, e.g., "the lack of systematic and effective treatment methods"), and (4) environmental stressors of COVID-19 (4 items, e.g., "the stability/instability of my family's financial situation"), $\alpha=0.957$. Participants rated each item on a 5-point Likert scale ranging from 1 (no stress) to 5 (great stress). Higher total scores indicate greater levels of COVID19-related stress.

\section{Rumination}

The Chinese Version of the Ruminative Responses Scale [39], adapted from [40], was administered to measure participants' level of rumination during the past month. The scale includes three dimensions: (1) compulsive thinking (5 items, e.g., "I think 'What am I doing to deserve this?'”), (2) reflective thinking (5 items, e.g., "I go someplace alone to think about my feelings"), and (3) symptomatic rumination (12 items, e.g., "I think 'I won't be able to do my job if I don't snap out of this'"), $\alpha=0.963$. Participants rated each item on a 4-point Likert scale ranging from 1 (never) to 4 (always), with higher total scores indicating higher levels of rumination. This scale has been used in the Chinese population $[21,26]$ with good reliability and validity.

\section{Poor Sleep Quality}

The Chinese Version of the Pittsburg Sleep Quality Index [41], adapted from [42], was used to evaluate participants' sleep quality during the past month. The scale contains 19 items that cover seven equally weighted dimensions of sleep quality: (1) subjective sleep quality (1 item, e.g., "how would you rate your sleep quality overall"), (2) sleep latency (2 items, e.g., "how long has it usually take you to fall asleep each night"), (3) sleep duration (1 item, e.g., "how many hours of actual sleep did you get at night?"), (4) habitual sleep efficiency ( 3 items, e.g., "when have you usually gone to bed at night"), (5) sleep disturbances (9 items, e.g., "how often have you had trouble sleeping because you cannot get to sleep within 30 min"), (6) use of sleep medication (1 item, e.g., "how often have you taken medicine to help you sleep"), and (7) daytime dysfunction (2 items, e.g., "how 


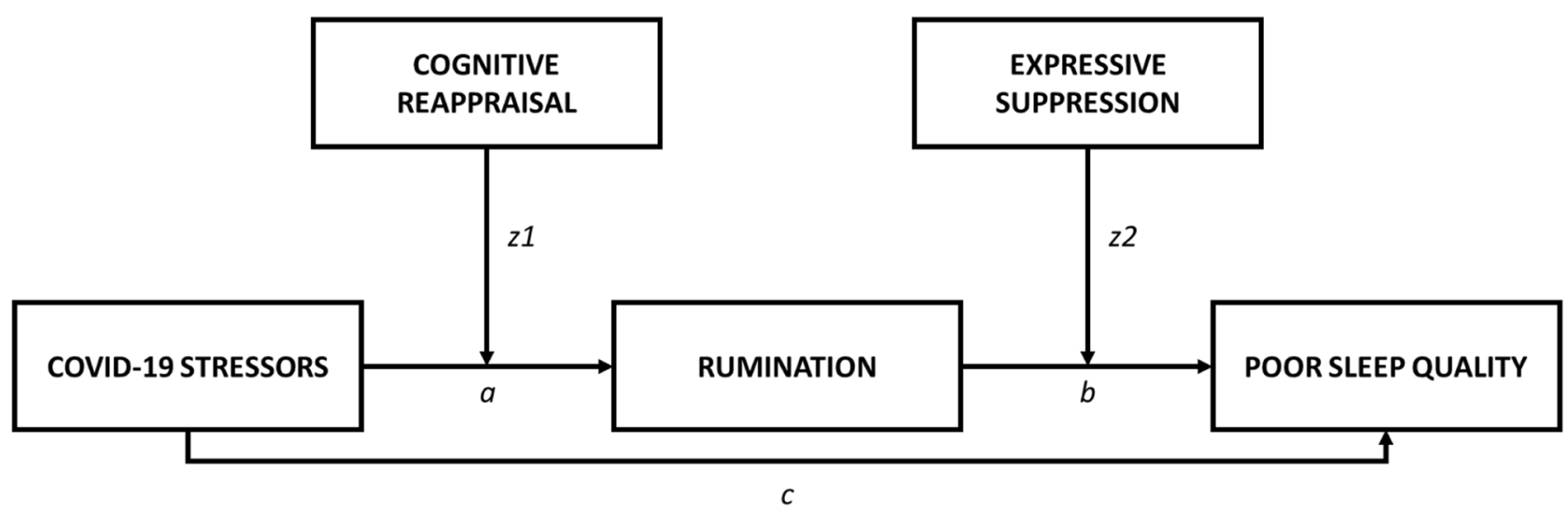

Fig. 1 The proposed moderated mediation model

much of a problem has it been for you to keep up enough enthusiasm to get things done"), $\alpha=0.869$. Following procedures from a previous study [43], scores of each dimension were averaged to produce the final score, with higher scores indicating poorer sleep quality. All items were rated on a 4-point Likert scale ranging from 0 (high quality of sleep) to 3 (low quality of sleep). This scale has been used with good reliability in the Chinese population in prior research [43].

\section{Emotion Regulation}

The Emotion Regulation Questionnaire was used to assess students' emotion regulation strategy in its Chinese version [44], adapted from [45]. This scale consists of two dimensions: (1) cognitive reappraisal (6 items, e.g., "I control my emotions by changing the way I think about the situation I' $m$ in," $\alpha=0.932$ ) and (2) expressive suppression (4 items, e.g., "I control my emotions by not expressing them," $\alpha=0.826$ ). All items were rated on a 7-point Likert scale ranging from 1 (strongly disagree) to 7 (strongly agree). The higher scores on each subscale indicate the higher frequency with which college students use cognitive reappraisal or expressive suppression. Good reliability and validity of the scale were shown in the Chinese population [46, 47].

\section{Analytical Strategy}

Skewness and kurtosis values [48] were within the acceptable ranges (i.e., skewness ranged from -0.40 to 1.44 and kurtosis ranged from -0.62 to 2.34 ). Gender (dummy coded as 0 [female $]$ and 1 [male]) was controlled for in the subsequent analyses based on prior findings whereby women tended to report experiencing more sleep problems than men [49-51]. The mediating effect of rumination was tested using the PROCESS macro for SPSS, Model 4, while the moderated mediation effect was constructed through Model 21 [52]. Simple slope tests were performed to demonstrate the interaction patterns, and significant moderation effects were probed at plus and minus one standard deviation from the mean of the predictor variables. All study variables were standardized by calculating $z$-scores, and the mediation and moderated mediation models utilized 5000 bootstrapped resamples to calculate confidence intervals [52] (Fig. 1).

\section{Results}

\section{Preliminary Analysis}

The means, standard deviations, and bivariate correlations of the study variables are shown in Table 1. Participant
Table 1 Descriptive statistics and bivariate correlations of study variables

\begin{tabular}{lllllll}
\hline Variable & $\boldsymbol{M}$ & $\boldsymbol{S D}$ & $\mathbf{1}$ & $\mathbf{2}$ & $\mathbf{3}$ & $\mathbf{4}$ \\
\hline 1. COVID-19 stressors & 2.29 & 0.84 & - & & \\
2. Rumination & 1.57 & 0.55 & $.566^{* * *}$ & - & \\
3. Poor sleep quality & 0.79 & 0.55 & $.431^{* * *}$ & $.604^{* * *}$ & - & \\
4. Cognitive reappraisal & 3.57 & 1.29 & $-.164^{* * *}$ & $-.142^{* * *}$ & $.098^{* * *}$ & - \\
5. Expressive suppression & 3.68 & 1.20 & $.237^{* * *}$ & $.273^{* * *}$ & $.223^{* * *}$ & $-.565^{* * *}$ \\
\hline
\end{tabular}

${ }^{*} p<.05,{ }^{* *} p<.01,{ }^{* * *} p<.001$ 
Table 2 Regression analyses for each path of the conceptual model

\begin{tabular}{|c|c|c|c|c|c|c|c|c|c|c|c|c|}
\hline \multirow[t]{3}{*}{ Predictor } & \multicolumn{3}{|c|}{$\begin{array}{l}\text { Criterion variable Rumination } \\
\text { (a) }\end{array}$} & \multicolumn{3}{|c|}{$\begin{array}{l}\text { Criterion variable } \\
\text { Poor sleep quality }(b / c)\end{array}$} & \multicolumn{3}{|c|}{$\begin{array}{l}\text { Criterion variable } \\
\text { Rumination }\left(z_{1}\right)\end{array}$} & \multicolumn{3}{|c|}{$\begin{array}{l}\text { Criterion variable } \\
\text { Poor sleep quality }\left(z_{2}\right)\end{array}$} \\
\hline & \multirow[b]{2}{*}{$\beta$} & \multicolumn{2}{|l|}{$95 \% \mathrm{CI}$} & \multirow[b]{2}{*}{$\beta$} & \multicolumn{2}{|c|}{ 95\% CI } & \multirow[b]{2}{*}{$\beta$} & \multicolumn{2}{|c|}{ 95\% CI } & \multirow[b]{2}{*}{$\beta$} & \multicolumn{2}{|l|}{ 95\% CI } \\
\hline & & Lower & Upper & & Lower & Upper & & Lower & Upper & & Lower & Upper \\
\hline Gender $(1=$ male $)$ & $-.105^{*}$ & -.204 & -.006 & .090 & -.005 & .185 & $-.133^{* * *}$ & -.224 & -.033 & $.096^{*}$ & -.002 & .191 \\
\hline $\mathrm{CS}$ & $.572^{* * *}$ & .523 & .621 & $.125^{* * * *}$ & .068 & .182 & $.561^{* * *}$ & .512 & .610 & $.126^{* * *}$ & .068 & .183 \\
\hline Rumination & & & & $.533^{* * *}$ & .476 & .590 & & & & $.497^{* * *}$ & .436 & .557 \\
\hline CR & & & & & & & $-.092^{* *}$ & -.147 & -.036 & & & \\
\hline $\mathrm{CS} \times \mathrm{CR}$ & & & & & & & $-.053^{*}$ & -.097 & -.008 & & & \\
\hline ES & & & & & & & & & & $.065^{*}$ & .015 & .114 \\
\hline Rumination $\times \mathrm{ES}$ & & & & & & & & & & $.053^{* *}$ & .013 & .093 \\
\hline$R^{2}$ & .323 & & & .378 & & & .330 & & & .385 & & \\
\hline$F$ & $262.928^{* * * *}$ & & & 223.544 & & & $135.505^{* * *}$ & & & $137.452^{* * *}$ & & \\
\hline
\end{tabular}

CS COVID-19 stressors, $C R$ cognitive reappraisal, ES expressive suppression. Gender was dummy coded. Specific paths corresponding to conceptual model given in parentheses. ${ }^{*} p<.05,{ }^{* *} p<.01,{ }^{* * *} p<.001$

responses to COVID-19 stressors ranged from the lowest to highest possible scores (i.e., 1 to 5 ), and $44.12 \%$ of them reported COVID-19 stress levels that were above the midpoint value of the scale. The proportion of students who met the criteria of poor sleep quality [42] was $37.7 \%$, and those who reported above the midpoint value of the rumination scale was $18.63 \%$.

\section{Mediation Effect Analysis}

COVID-19 stressors were positively correlated with rumination $(\beta=0.572, p<0.001)$ and poor sleep quality $(\beta=0.125$, $p<0.001)$. Rumination was positively associated with poor sleep quality $(\beta=0.533, p<0.001)$. The direct effect of COVID-19 stressor on poor sleep quality remained positive. Accordingly, rumination partially mediated the effect of COVID-19 stressors on poor sleep quality (indirect effect $=0.305, \mathrm{SE}=0.032,95 \% \mathrm{CI}_{\text {boot }}=[0.244,0.369]$ ), accounting for $70.93 \%$ of the total effect (Table 2). Therefore, hypothesis 1 was supported.

\section{Moderated Mediation Effect Analysis}

Cognitive reappraisal moderated the effect of COVID-19 stressors on rumination ( $\beta=-0.053, p=0.020$; Fig. 2 ). Rumination levels by COVID-19 stressors, separately for low (i.e., one $S D$ below the mean) and high (i.e., one $S D$ above the mean) degrees of cognitive reappraisal, are presented in Fig. 3a. COVID-19 stressors were positively associated with rumination for both students with low $\left(\beta_{\text {simple }}=0.614\right.$,

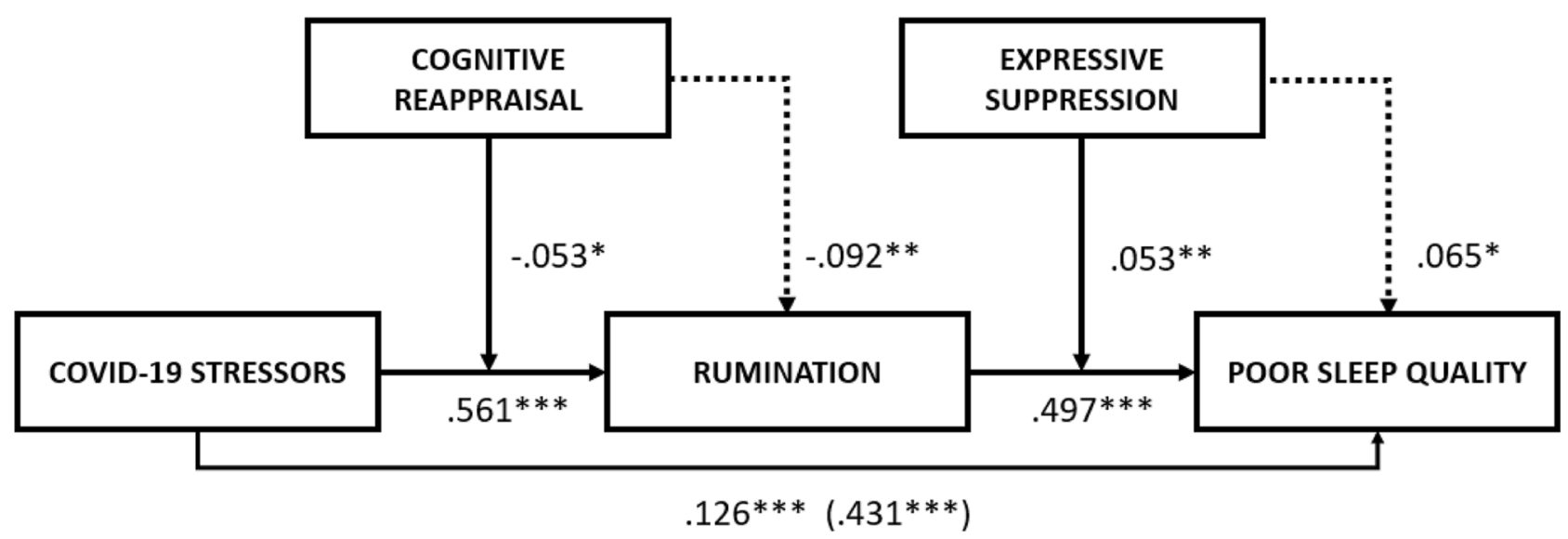

Fig. 2 Moderated mediation model. ${ }^{*} p<.05,{ }^{* *} p<.01, * * * p<.001$. Dotted lines represent direct paths from the moderators to the mediator and outcome variables. Standardized beta coefficients are presented; total effect is presented in the parentheses 
(A) $\mathrm{CS} \times \mathrm{CR}$

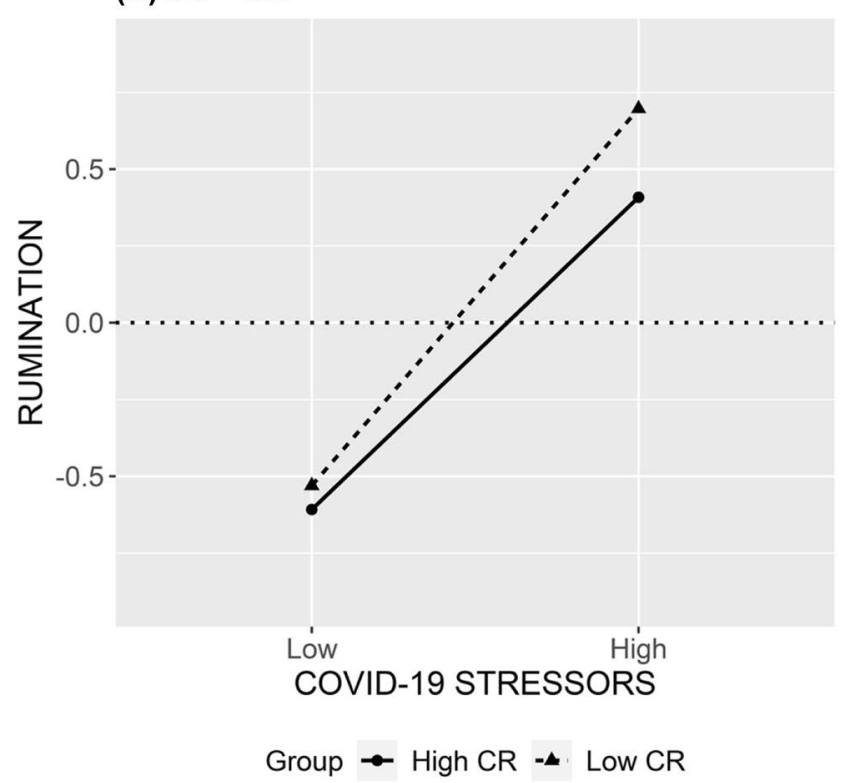

(B) RUMINATION × ES

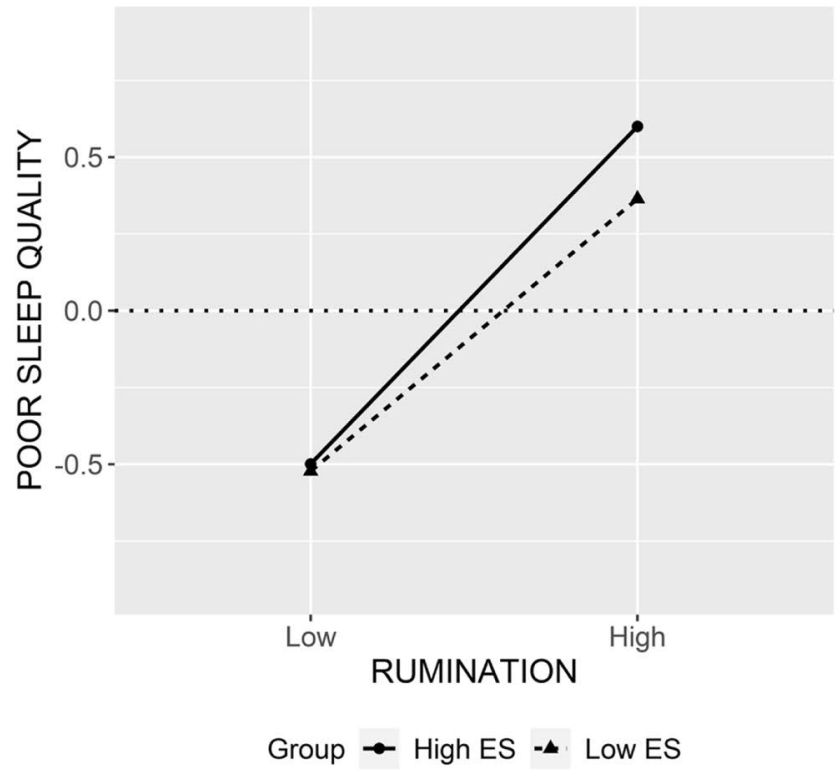

Fig. 3 Moderated paths. High and low values indicate +1 SD and - 1 SD from the mean, respectively. CS COVID-19 stressors, CR cognitive reappraisal, $E S$ expressive suppression. $Y$ axes represent beta-coefficients of the interaction variables predicting $y$-axis label variable

$p<0.001,95 \% \mathrm{CI}[0.549,0.679])$ and high levels of cognitive reappraisal $\left(\beta_{\text {simple }}=0.508, p<0.001,95 \%\right.$ CI $[0.441$, $0.576])$ but the correlation was notably weaker in the latter.

Expressive suppression moderated the effect of rumination on poor sleep quality ( $\beta=0.053, p=0.014$; Fig. 2$)$. Poor sleep quality by rumination, separately for low (i.e., one $S D$ below the mean) and high (i.e., one $S D$ above the mean) levels of expressive suppression, are given in Fig. 3b. Rumination was positively associated with poor sleep quality for students with both low $\left(\beta_{\text {simple }}=0.444\right.$, $p<0.001,95 \%$ CI $[0.361,0.526])$ and high levels of expressive suppression ( $\beta_{\text {simple }}=0.550, p<0.001,95 \%$ CI $[0.489,0.611])$, but the correlation was notably stronger in the latter.

The bias-corrected percentile bootstrap analysis further indicated that cognitive reappraisal and expressive suppression moderated the mediating effect. For college students with low cognitive reappraisal, the indirect effect of rumination on the COVID-19 stressors and poor sleep quality association was stronger $(\beta=0.305$, $\left.\mathrm{SE}=0.035,95 \% \mathrm{CI}_{\text {boot }}=[0.238,0.375]\right)$ than those with high cognitive reappraisal $(\beta=0.252, \mathrm{SE}=0.027,95 \%$ $\left.\mathrm{CI}_{\text {boot }}=[0.204,0.308]\right)$. The standardized moderation effect of cognitive reappraisal was 0.003 . Furthermore, for college students with low expressive suppression, the indirect effect of rumination was weaker $(\beta=0.249$, $\left.\mathrm{SE}=0.029,95 \% \mathrm{CI}_{\text {boot }}=[0.195,0.311]\right)$ compared to those high in expressive suppression $(\beta=0.308, \mathrm{SE}=0.037$,
95\% $\left.\mathrm{CI}_{\text {boot }}=[0.235,0.383]\right)$. The standardized moderation effect of expressive suppression was 0.004. Hence, Hypotheses 2 and 3 were supported.

\section{Discussion}

This study found that COVID-19 stressors were significantly linked to poor sleep quality, and rumination partially mediated this association among Chinese college students. Furthermore, the indirect relation between COVID-19 stressors and poor sleep quality through rumination was moderated by cognitive reappraisal and expression suppression. The strength of the association between COVID-19 stressors and rumination was weaker among those who tended to cognitively reappraise, and the strength of the association between rumination and poor sleep quality was stronger among those who tended to suppress their emotion expression.

\section{The Mediating Role of Rumination}

The finding that rumination functions as one mediating mechanism linking stress to poor sleep quality was consistent with previous studies $[13,25,53]$. This further evidences that ruminative behavior and thinking can emerge 
in the presence of uncontrollable factors such as unprecedented and repetitive COVID-19 stressors [17] and that perseverative cognition can be health-threatening due to its function to sleep quality [22].

COVID-19 stressors remained a direct correlate of poor sleep quality, suggesting that COVID-19 stress is likely a unique and salient risk factor for poor sleep quality. Indeed, one prior study had found that daily consumption of COVID-19 news and media was associated with depression and poor sleep quality, similar to the current finding where media exposure to COVID-19 was one component of stressors [54]. Further, loneliness has been resulted from the pandemic, especially among college students who may feel that they are unable to experience a significant part of their social development due to the public health measures (e.g., lockdowns) [55-57]. As partially captured in our COVID-19 stressors scale, loneliness may likely disrupt young adults' sleep and induce greater negative outcomes in the long run [58]. Hence, in addition to paying attention to the rumination behavior, social interventions are equally necessary to target COVID-19 stressors themselves to mitigate college students' susceptibility to poor sleep quality.

Additionally, there could be other factors serving as mechanisms linking COVID-19 stressors and poor sleep quality. Behavioral outcomes due to the pandemic and the stress associated with them (i.e., increased screen time, daytime napping) could influence circadian rhythm, stages of sleep (e.g., slow-wave sleep), and ultimately sleep quality $[59,60]$. Thus, further research is essential to investigate other relevant mechanisms underlying the direct association between COVID-19 stressors and sleep quality.

\section{The Moderating Role of Emotion Regulation}

The association between COVID-19 stressors and rumination became weaker as the students' tendency to cognitively reappraise increased. This finding is consistent with the risk buffering model [61] which suggests that cognitive reappraisal can serve as a protective factor in mitigating the adverse effects of psychological distress. Specifically, students who routinely engage in cognitive reappraisal may have been able to apply attention distribution away from negative events $[62,63]$. For instance, students adept at cognitive reappraisal might be more readily capable of directing their attention toward the positive elements of the pandemic, such as by strengthening social bonds through solidarity [56]. Thus, even when experiencing COVID-19 stressors, these students might be less likely to suffer from adverse outcomes of stress (i.e., poor sleep quality) than those who are less efficient in cognitive reappraisal.
The association between rumination and poor sleep quality grew stronger as expressive suppression also increased. This result lends credence to prior literature whereby expressive suppression promoted sympathetic activation of the cardiovascular systems [64], which could, in turn, worsen sleep quality (e.g., REM sleep) [65, 66]. Furthermore, for students with high levels of expressive suppression, when they have not only repeated negative thoughts about COVID19 , but also difficulties uttering their feelings appropriately, they could experience a cumulative effects of the negative emotions on health consequences [28]. Thus, individuals who have high levels of expressive suppression are likely to experience a stronger correlation between rumination and impaired sleep in the current research.

\section{Limitations}

Interpretation of the findings of this study must consider the following limitations. First, the current study was crosssectional and thus causal inferences are limited. The mediation model, which assumes underlying causality [67], should be replicated in future studies with experimental and longitudinal designs and physiological measurements. However, given the theory and empirical evidence that indicate rumination could mediate the effect of stress on health outcomes [17, 68], including sleep health [53, 69], and that cross-sectional studies could provide valuable information to reveal the associations between variables [48, 52], we are reasonably confident in conceptual sequence and the use of the mediation model in the present study [70]. Secondly, our sample consisted of college students in China, and the dataset only contained respondents who have completed all measurement items. Thus, the generalizability of the findings is constrained to the social context of the study. Third, the current model examined rumination as a mediating mechanism and did not consider other factors that may be at play. Lastly, although participants were assured of their anonymity, all variables were assessed via self-report measures and may have been subject to the social-desirability bias.

\section{Conclusion}

This study unpacked how COVID-19 stressors related to sleep health. Given that poor sleep quality is associated with maladaptive psychological, physiological, and achievement outcomes to college students, our study represents a crucial step in understanding the sleep health issue among Chinese college students amidst the COVID-19 pandemic. Further, the study highlighted the roles COVID-19 stressors, rumination, and emotion regulation play in sleep quality. Interventions that address rumination and appropriate emotion 
regulation strategies might prove efficacious in improving sleep quality among college students during the pandemic. As college campuses begin reopening despite the lingering threats of COVID-19 to public health, college mental health professionals should pay special attention to students' sleep quality. Additionally, it is equally important to actively promote student's understanding of COVID-19 related stressors, foster proper emotion regulation strategies, and encourage help-seeking.

Funding This study was funded by the Jiangxi' Educational Scientific Planning Project (20YB029), Jiangxi' Key Research Base Project of Humanities and Social Sciences (JD20068), and Science and Technology Research Project of Jiangxi' Department of Education (GJJ200306).

\section{Declarations}

Ethical Approval All procedures performed in studies involving human participants were in accordance with the ethical standards of the institutional and/or national research committee and with the 1964 Helsinki declaration and its later amendments or comparable ethical standards.

Informed Consent Informed consent was obtained from all individual participants included in the study.

Conflict of Interest The authors declare no competing interests.

\section{References}

1. Altena E, Baglioni C, Espie CA, et al. Dealing with sleep problems during home confinement due to the COVID-19 outbreak: Practical recommendations from a task force of the european cbt-i academy. J Sleep Res. 2020;29: e13052. https://doi.org/10.1111/ jsr.13052.

2. Casagrande M, Favieri F, Tambelli R, Forte G. The enemy who sealed the world: Effects quarantine due to the COVID-19 on sleep quality, anxiety, and psychological distress in the italian population. Sleep Med. 2020;75:12-20. https://doi.org/10.1016/j. sleep.2020.05.011.

3. Huang Y, Zhao N. Mental health burden for the public affected by the COVID-19 outbreak in china: Who will be the high-risk group? Psychol Health Med. 2021;26:23-34. https://doi.org/10. 1080/13548506.2020.1754438.

4. Lin LY, Wang J, Ou-Yang XY, et al. The immediate impact of the 2019 novel coronavirus (COVID-19) outbreak on subjective sleep status. Sleep Med. 2021;77:348-54. https://doi.org/10.1016/j. sleep.2020.05.018.

5. Xiao H, Zhang Y, Kong D, Li S, Yang N. The effects of social support on sleep quality of medical staff treating patients with coronavirus disease 2019 (COVID-19) in january and february 2020 in China. Med Sci Monit. 2020;26:e923549-51.

6. Fu W, Wang C, Zou L, et al. Psychological health, sleep quality, and coping styles to stress facing the COVID-19 in Wuhan. China Transl Psychiatry. 2020;10:225. https://doi.org/10.1038/ s41398-020-00913-3.

7. Mandelkorn U, Genzer S, Choshen-Hillel S, et al. Escalation of sleep disturbances amid the COVID-19 pandemic: A cross-sectional international study. J Clin Sleep Med. 2021;17:4553. https://doi.org/10.5664/jcsm.8800.

8. Alfano CA, Zakem AH, Costa NM, Taylor LK, Weems CF. Sleep problems and their relation to cognitive factors, anxiety, and depressive symptoms in children and adolescents. Depress Anxiety. 2009;26:503-12. https://doi.org/10.1002/da.20443.

9. Curcio G, Ferrara M, De Gennaro L. Sleep loss, learning capacity and academic performance. Sleep Med Rev. 2006;10:323-37. https://doi.org/10.1016/j.smrv.2005.11.001.

10. Pilcher JJ, Ginter DR, Sadowsky B. Sleep quality versus sleep quantity: Relationships between sleep and measures of health, well-being and sleepiness in college students. J Psychosom Res. 1997;42:583-96. https://doi.org/10.1016/S0022-3999(97) 00004-4

11. Shin JE, Kim JK. How a good sleep predicts life satisfaction: The role of zero-sum beliefs about happiness. Front Psychol. 2018;9. https://doi.org/10.3389/fpsyg.2018.01589.

12. Lund HG, Reider BD, Whiting AB, Prichard JR. Sleep patterns and predictors of disturbed sleep in a large population of college students. J Adolesc Health. 2010;46:124-32.

13. Ye B, Wu D, Im H, Liu M, Wang X, Yang Q. Stressors of COVID19 and stress consequences: The mediating role of rumination and the moderating role of psychological support. Child Youth Serv Rev. 2020;118: 105466. https://doi.org/10.1016/j.childyouth.2020. 105466.

14. Ye B, Zhou X, Im H, Liu M, Wang XQ, Yang Q. Epidemic rumination and resilience on college students' depressive symptoms during the COVID-19 pandemic: The mediating role of fatigue. Frontiers in Public Health. 2020;8. https://doi.org/10.3389/fpubh. 2020.560983.

15. Copeland WE, McGinnis E, Bai Y, et al. Impact of COVID-19 pandemic on college student mental health and wellness. J Am Acad Child Adolesc Psychiatry. 2021;60:134-41. e2.

16. McGinty EE, Presskreischer R, Anderson KE, Han H, Barry CL. Psychological distress and COVID-19-related stressors reported in a longitudinal cohort of us adults in April and July 2020. JAMA. 2020;324:2555-7.

17. Nolen-Hoeksema S, Wisco BE, Lyubomirsky S. Rethinking rumination. Perspect Psychol Sci. 2008;3:400-24. https://doi.org/10. 1111/j.1745-6924.2008.00088.x.

18. Smyth J, Zawadzki M, Gerin W. Stress and disease: A structural and functional analysis. Soc Personal Psychol Compass. 2013;7:217-27. https://doi.org/10.1111/spc3.12020.

19. Robinson MS, Alloy LB. Negative cognitive styles and stressreactive rumination interact to predict depression: A prospective study. Cognit Ther Res. 2003;27:275-91. https://doi.org/10. 1023/A:1023914416469.

20. Brosschot JF, Gerin W, Thayer JF. The perseverative cognition hypothesis: A review of worry, prolonged stress-related physiological activation, and health. J Psychosom Res. 2006;60:113-24. https://doi.org/10.1016/j.jpsychores.2005.06.074.

21. Chen X, Huang Y. Study on relationship between self-identity, peer stress and rumination thinking in college students. Occupation and Health. 2019;35:2115-9. https://doi.org/10.13329/j.cnki. zyyjk.2019.0557.

22. Bian X-H, Hou XL, Zuo ZH, et al. Depression and sleep quality among chinese college students: The roles of rumination and self-compassion. Curr Psychol. 2020. https://doi.org/10.1007/ s12144-020-00946-3.

23. Thomsen DK, Yung Mehlsen M, Christensen S, Zachariae R. Rumination-relationship with negative mood and sleep quality. Pers Individ Dif. 2003;34:1293-301. https://doi.org/10.1016/ S0191-8869(02)00120-4.

24. Guastella AJ, Moulds ML. The impact of rumination on sleep quality following a stressful life event. Pers Individ Dif. 2007;42:1151-62. https://doi.org/10.1016/j.paid.2006.04.028. 
25. Zawadzki MJ, Graham JE, Gerin W. Rumination and anxiety mediate the effect of loneliness on depressed mood and sleep quality in college students. Health Psychol. 2013;32:212-22. https:// doi.org/10.1037/a0029007.

26. Wang $\mathrm{T}, \mathrm{Xu} \mathrm{X}$, Wang $\mathrm{H}$, et al. Relationship between trait anxiety and positive and negative affect in young male military personnel: Roles of rumination and educational level. Journal of Third Military Medical University. 2020;42:119-24. https://doi.org/10. 16016/j.1000-5404.201908051.

27. Troy AS, Wilhelm FH, Shallcross AJ, Mauss IB. Seeing the silver lining: Cognitive reappraisal ability moderates the relationship between stress and depressive symptoms. Emotion. 2010;10:78395. https://doi.org/10.1037/a0020262.

28. Gross JJ. Emotion regulation: Current status and future prospects. Psychol Inq. 2015;26:1-26. https://doi.org/10.1080/1047840X. 2014.940781.

29. John OP, Gross JJ. Individual differences in emotion regulation. Handbook of emotion regulation: The Guilford Press; 2007. p. 351-72.

30. Gross JJ. Emotion regulation: Affective, cognitive, and social consequences. Psychophysiology. 2002;39:281-91. https://doi.org/10. 1017/S0048577201393198.

31. Lazarus RS, Folkman S. Stress, appraisal, and coping: Springer publishing company. 1984.

32. Ortony A, Clore GL, Collins A. The cognitive structure of emotions: Cambridge university press. 1990.

33. Moore SA, Zoellner LA, Mollenholt N. Are expressive suppression and cognitive reappraisal associated with stress-related symptoms? Behav Res Ther. 2008;46:993-1000.

34. Lee C-YS, Goldstein SE. Loneliness, stress, and social support in young adulthood: Does the source of support matter? J Youth Adolesc. 2016;45:568-80. https://doi.org/10.1007/ s10964-015-0395-9.

35. Quick JC, Henderson DF. Occupational stress: Preventing suffering, enhancing wellbeing. Int J Environ Res Public Health. 2016;13:459.

36. Cohen S, Wills TA. Stress, social support, and the buffering hypothesis. Psychol Bull. 1985;98:310-57. https://doi.org/10. 1037/0033-2909.98.2.310.

37. Latif I, Hughes ATL, Bendall RCA. Positive and negative affect mediate the influences of a maladaptive emotion regulation strategy on sleep quality. Frontiers in Psychiatry. 2019;10. https://doi. org/10.3389/fpsyt.2019.00628.

38. Tang NK, Harvey AG. Effects of cognitive arousal and physiological arousal on sleep perception. Sleep. 2004;27:69-78. https://doi. org/10.1093/sleep/27.1.69.

39. Han X, Yang HF. Chinese version of nolen-hoeksema ruminative responses scale (rrs) used in 912 college students: Reliability and validity [chinese version of nolen-hoeksema ruminative responses scale (rrs) used in 912 college students: Reliability and validity.]. Chin J Clin Psychol. 2009;17:550-1.

40. Nolen-Hoeksema S, Morrow J. A prospective study of depression and posttraumatic stress symptoms after a natural disaster: The 1989 loma prieta earthquake. J Pers Soc Psychol. 1991;61:11521. https://doi.org/10.1037/0022-3514.61.1.115.

41. Liu X, Tang M, Hu L. Reliability and validity of the pittsburgh sleep quality index. Chinese journal of psychiatry. 1996;29:103-7.

42. Buysse DJ, Reynolds CF, Monk TH, Berman SR, Kupfer DJ. The pittsburgh sleep quality index: A new instrument for psychiatric practice and research. Psychiatry Res. 1989;28:193-213. https:// doi.org/10.1016/0165-1781(89)90047-4.

43. Zhang D, Hu X, Liu Q. Stress and sleep quality among undergraduate students: chain mediating effects of rumination and resilience. Journal of Psychological Science. 2021;44:90-6. https:// doi.org/10.16719/j.cnki.1671-6981.20210113.
44. Wang L, Liu H, Li Z, Du W. Reliability and validity of emotion regulation questionnaire chinese revised version. Chin J Health Psychol. 2007;15:503-5. https://doi.org/10.3969/j.issn.10051252.2007.06.034.

45. Gross JJ, John OP. Individual differences in two emotion regulation processes: Implications for affect, relationships, and wellbeing. J Pers Soc Psychol. 2003;85:348-62. https://doi.org/10. 1037/0022-3514.85.2.348.

46. Sun Y, Bo S, Lv J. Brain network analysis of cognitive reappraisal and expressive inhibition strategies: Evidence from eeg and erp. Acta Psychol Sin. 2019;52:12-25.

47. Tian $\mathrm{Y}, \mathrm{Wu} \mathrm{X}$, Wang $\mathrm{W}, \mathrm{Z}$ hou $\mathrm{X}$. The relation between attachment and $\mathrm{ptsd} / \mathrm{ptg}$ among adolescents after the wenchuan earthquake: The mediating roles of cognitive reappraisal and expressive suppression. Psychol Dev Educ. 2018;34:105-11. https://doi.org/10. 16187/j.cnki.issn1001-4918.2018.01.13.

48. Kline RB. Principles and practice of structural equation modeling: Guilford publications. 2015.

49. Jiang M, Zhao Y, Wang Y, et al. The status of college students' sleep quality and its relationship with internet addiction, anxiety and depression. 2019.

50. Chow CM, Homa J, Amersdorfer A. Gender differences in sleep problems: The mediating role of co-rumination and depressive symptoms. Pers Individ Dif. 2017;108:10-3.

51. Glavin EE, Matthew J, Spaeth AM. Gender differences in the relationship between exercise, sleep, and mood in young adults. Health Educ Behav. 2021:1090198120986782.

52. Hayes AF. Introduction to mediation, moderation, and conditional process analysis: A regression-based approach: Guilford publications. 2017.

53. Berset M, Elfering A, Lüthy S, Lüthi S, Semmer NK. Work stressors and impaired sleep: Rumination as a mediator. Stress Health. 2011;27:e71-82. https://doi.org/10.1002/smi.1337.

54. Holman EA, Thompson RR, Garfin DR, Silver RC. The unfolding COVID-19 pandemic: A probability-based, nationally representative study of mental health in the united states. Sci Adv. 2020;6:eabd5390. https://doi.org/10.1126/sciadv.abd5390.

55. Li LZ, Wang S. Prevalence and predictors of general psychiatric disorders and loneliness during COVID-19 in the united kingdom. Psychiatry Res. 2020;291: 113267. https://doi.org/10. 1016/j.psychres.2020.113267.

56. Vaterlaus JM, Shaffer T, Pulsipher L. College student interpersonal and institutional relationships during the COVID-19 pandemic: A qualitative exploratory study. Soc Sci J. 2021:1-14. https://doi.org/10.1080/03623319.2021.1949553.

57. Killgore WDS, Cloonan SA, Taylor EC, Dailey NS. Loneliness: A signature mental health concern in the era of COVID-19. Psychiatry Res. 2020;290: 113117. https://doi.org/10.1016/j.psychres. 2020.113117.

58. Matthews T, Danese A, Gregory AM, Caspi A, Moffitt TE, Arseneault L. Sleeping with one eye open: Loneliness and sleep quality in young adults. Psychol Med. 2017;47:2177-86. https:// doi.org/10.1017/S0033291717000629.

59. Gupta R, Grover S, Basu A, et al. Changes in sleep pattern and sleep quality during COVID-19 lockdown. Indian J Psychiatry. 2020;62:370-8. https://doi.org/10.4103/psychiatry. IndianJPsychiatry_523_20.

60. Majumdar P, Biswas A, Sahu S. COVID-19 pandemic and lockdown: Cause of sleep disruption, depression, somatic pain, and increased screen exposure of office workers and students of india. Chronobiol Int. 2020;37:1191-200. https://doi.org/10.1080/ 07420528.2020 .1786107$.

61. Masten AS. Ordinary magic: Resilience processes in development. Am Psychol. 2001;56:227-38. https://doi.org/10.1037/ 0003-066X.56.3.227. 
62. Gross JJ, Thompson RA. Emotion regulation: Conceptual foundations. Handbook of emotion regulation. New York, NY, US: The Guilford Press. 2007;3-24.

63. Koole SL. The psychology of emotion regulation: An integrative review. Cogn Emot. 2009;23:4-41. https://doi.org/10.1080/ 02699930802619031.

64. Gross JJ. Antecedent- and response-focused emotion regulation: Divergent consequences for experience, expression, and physiology. J Pers Soc Psychol. 1998;74:224-37. https://doi.org/10. 1037//0022-3514.74.1.224.

65. Seravalle G, Mancia G, Grassi G. Sympathetic nervous system, sleep, and hypertension. Curr Hypertens Rep. 2018;20:74. https:// doi.org/10.1007/s11906-018-0874-y.

66. Somers VK, Dyken ME, Mark AL, Abboud FM. Sympatheticnerve activity during sleep in normal subjects. N Engl J Med. 1993;328:303-7. https://doi.org/10.1056/nejm199302043280502.
67. O'Laughlin KD, Martin MJ, Ferrer E. Cross-sectional analysis of longitudinal mediation processes. Multivar Behav Res. 2018;53:375-402.

68. Sarno EL, Newcomb ME, Mustanski B. Rumination longitudinally mediates the association of minority stress and depression in sexual and gender minority individuals. J Abnorm Psychol. 2020;129:355-63. https://doi.org/10.1037/abn0000508.

69. Liu L, Cao Q. Perceived stress and sleep quality among chinese drug users: Analysis of rumination as a mediator and resilience as a moderator. Int J Ment Health Addict. 2020:1-12.

70. Wen Z, Ye B. Analyses of mediating effects: The development of methods and models. Adv Psychol Sci. 2014;22:731.

Publisher's Note Springer Nature remains neutral with regard to jurisdictional claims in published maps and institutional affiliations. 